\title{
Design Strategy and Considerations to Improve Corona Discharge Breakdown in Groove Gap Waveguides
}

\author{
Aitor Morales-Hernández ${ }^{\# 1}$, Miguel Ferrando-Rocher ${ }^{\# 2}$, Miguel Á. Sánchez-Soriano ${ }^{\# 3}$, \\ Stephan Marini ${ }^{\# 4}$, Vicente E. Boria ${ }^{* 5}$ \\ \#Institute of Physics Applied to Sciences and Technologies, University of Alicante, Spain \\ \#Department of Physics, Systems Engineering and Signal Theory, University of Alicante, Spain \\ *iTEAM, Universitat Politècnica de València, Spain \\ $\left\{{ }^{1}\right.$ aitor.morales, ${ }^{2}$ miguel.ferrando, ${ }^{3}$ miguel.sanchez.soriano, ${ }^{4}$ s.marini $\} @$ ua.es
}

\begin{abstract}
This paper studies the corona discharge breakdown thresholds in groove gap waveguides, and proposes a design strategy to enhance its peak power handling capability (PPHC). The theoretical analysis is focused on the study of the quasi-TE 10 mode, and on the PPHC at different frequencies and multiple arrangements of the pin dimensions of the bed of nails. Next, the geometrical parameters width, length and separation of such pins are optimized for improving peak power limits. Finally, the simulated results show reasonably good performance with respect to the equivalent rectangular waveguide power limit.

Index Terms-Groove gap waveguide (GGW), corona dis-
\end{abstract} charge, peak power handling capability (PPHC), gas breakdown

\section{INTRODUCTION}

Corona discharge (also known as corona effect or gas breakdown) occurs when, in gas-filled components, an avalanche of electrons occurs [1]. The avalanche is created when the electrons impact the gas molecules, causing ionization. This leads to the appearance of an electronic plasma, which degrades the response of the component and can eventually destroy it.

The ionisation effect is a serious issue in both ground and satellite communications where moderate to high power signal levels are required. Due to the increasing problem of corona discharge in microwave devices, a big effort has been devoted in the last decade to understand, model and predict the occurrence of discharges for waveguide structures [2]-[4] and planar technology [5]-[7]. Therefore, it is important to determine which are the threshold RF power levels from which the corona effect arises. Fortunately, the recent appearance of powerful electromagnetic simulators has facilitated the study of the corona effect.

In this work, the corona discharge breakdown is quantified in groove gap waveguides (GGWs) at $\mathrm{Ku}$ - and $\mathrm{K}$-bands by using the SPARK3D ${ }^{\circledR}$ electromagnetic simulator, recently integrated in the CST Studio Suite software package. GGW is a technology that has demonstrated its potential to confine the field even in structures that are not sealed [8], [9]. Therefore, this paper studies the possible appearance of corona discharge breakdown in the air gaps typically presented in devices designed with GGW technology. Thus, the distribution of the quasi-TE 10 mode and the peak power handling capability
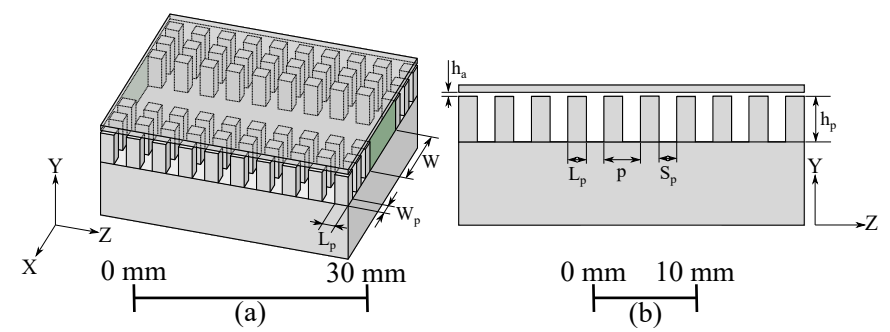

Fig. 1. Design for the theoretical analysis. (a) 3D view. (b) Lateral view.

(PPHC) are studied for GGW, and they will be compared to their rectangular waveguide (RW) counterparts. Finally, a design strategy that shows good results will be suggested for the improvement of corona discharge breakdown in GGW devices.

\section{TheOreticAl AnAlysis}

A generic GGW is commonly characterized by a periodic structure of a bed of nails, usually realized with square pins to ease the manufacturing process, which provides a stopband frequency response [10]. The main geometrical dimensions of the nails, i.e., $h_{a}, W_{p}, L_{p}, p$ and $h_{p}$ in Fig. 1 , determine the stopband behavior of the structure, so they must be properly designed for centering and adjusting the desired bandwidth. Some studies have already been realized for the study of the stopband properties, as can be found in [11].

The theoretical analysis presented in this section has been carried out for a generic GGW (see Fig. 1(a)), simulated by using the commercial software tool ANSYS HFSS ${ }^{1}$ for computing the electric fields inside the component. A rigorous study of how the geometrical dimensions of the pin lattice affect the distribution of the quasi-TE 10 mode and the PPHC will be described in what follows.

\section{A. Study of quasi-TE 10 mode distribution}

According to the results presented in [12], it is known that the field distribution in groove gap waveguides is similar to

${ }^{1}$ HFSS, Copyright (C) 2020 ANSYS, Inc. All rights reserved, available on https://www.ansys.com/products/electronics/ansys-hfss 


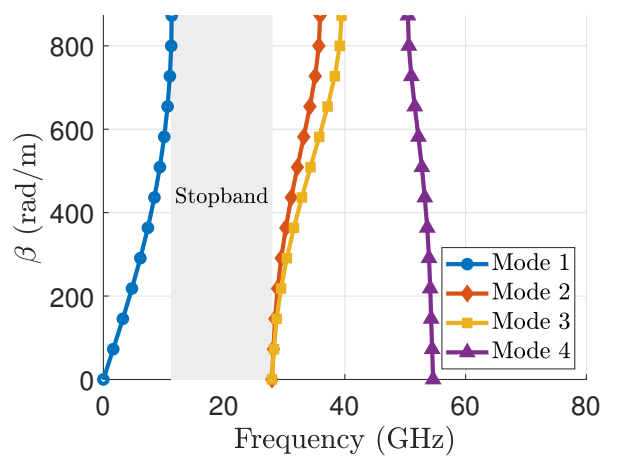

Fig. 2. GGW dispersion diagram: $p=3.60 \mathrm{~mm}, W_{p}=L_{p}=1.88 \mathrm{~mm}$, $h_{p}=4.50 \mathrm{~mm}$ and $h_{a}=0.40 \mathrm{~mm}$

the fundamental propagating $\mathrm{TE}_{10}$ mode in rectangular waveguides. However, some differences appear in the propagation characteristics as a function of the operation frequency.

To analyze the performance of the quasi- $\mathrm{TE}_{10}$ distribution, we firstly need to evaluate the stopband frequency range for a pin unit cell with the following dimensions by way of illustration: $p=3.60 \mathrm{~mm}, W_{p}=L_{p}=1.88 \mathrm{~mm}$, $h_{p}=4.50 \mathrm{~mm}$ and $h_{a}=0.40 \mathrm{~mm}$. The dispersion diagram obtained with $\mathrm{HFSS}^{1}$ eigenmode solver is shown in Fig. 2, where we can appreciate by a gray region a wide bandgap between $11.3 \mathrm{GHz}$ and $27.9 \mathrm{GHz}$, which covers the whole $\mathrm{Ku}$ - and K-bands, and where a quasi-TE 10 mode may be propagated.

In order to represent the quasi- $\mathrm{TE}_{10}$ mode distribution, a GGW as shown in Fig. 1(a) is designed and simulated with the foregoing pin dimensions and a selected value of $W=10.67 \mathrm{~mm}$, the latter equivalent to a WR-42 standard width $\left(f_{C_{T E_{10}}}=14.05 \mathrm{GHz}\right)$. In Fig. 3 the normalized $\left|\hat{E}_{y}\right|$ component for four frequencies is represented at two different heights, where the first plane is located at $y_{1}=h_{p}+h_{a} / 2$ (blue line) and the second plane is placed at $y_{2}=h_{p} / 2$ (red line), as specified in Fig. 3(a). As observed, for lower frequencies, $\left|\hat{E}_{y}\right|$ is higher above the first lateral row of pins; whereas for higher frequencies, the quasi- $\mathrm{TE}_{10}$ mode is better confined and the maximum electric field is located at the center of the waveguide section, similar to a $\mathrm{TE}_{10}^{R W}$ distribution.

Taking into account these results, when the operating frequency is close to the lower stopband limit, we could expect that the high interaction of $\left|\hat{E}_{y}\right|$ above the pins may strongly affect the PPHC of the device. Therefore, a design criterion for the pin dimensions would be important in order to adjust the stopband behavior to get a more confined quasi-TE 10 mode. Based on the foregoing, it becomes necessary to study in the following section the maximum peak power limit that GGWs can afford due to corona discharge breakdown. In Section III, a strategy will be proposed for improving PPHC in GGW technology.

\section{B. Study of Peak Power Handling Capability (PPHC)}

From the previous results, it is clear that secondary lobes of the quasi-TE 10 mode appear in the air gap above the first lateral row of pins for lower frequencies in the stopband region

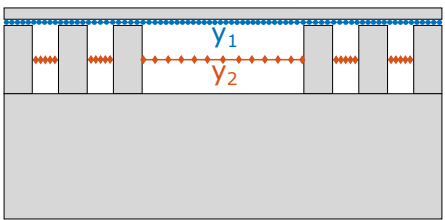

(a)
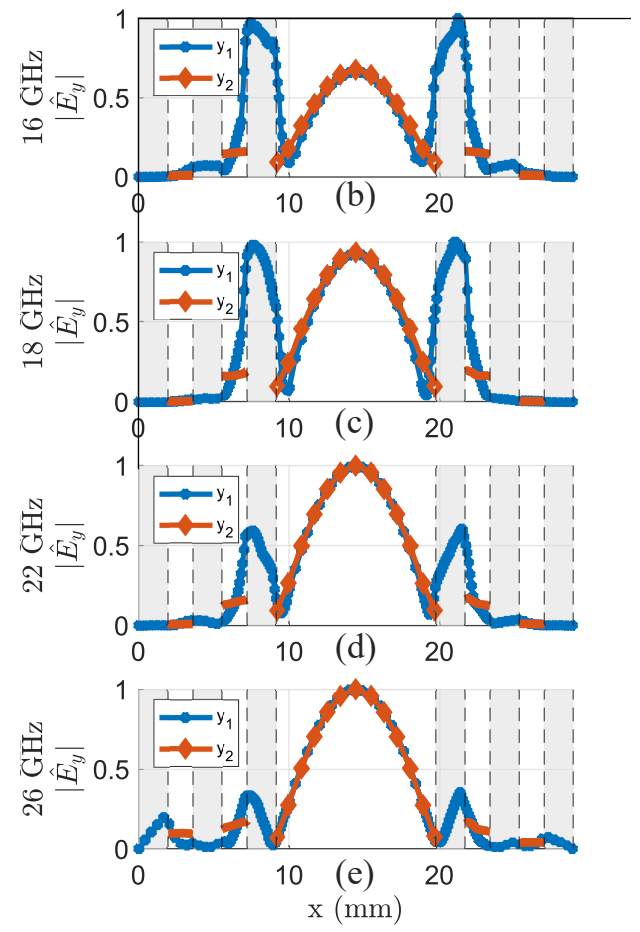

Fig. 3. $\left|\hat{E}_{y}(x)\right|$ at different frequencies and two height planes. (a) X-Y planes of the simulated GGW. (b) $16 \mathrm{GHz}$. (c) $18 \mathrm{GHz}$. (d) $22 \mathrm{GHz}$. (e) $26 \mathrm{GHz}$.

of Fig. 2. As a consequence, this high electric field may degrade the PPHC of the device, as it will be seen below.

For the study presented in this section, several layouts as shown in Fig. 1(a) have been designed for all the possible combinations of $h_{p}=\{3.00,4.50,6.00\} \mathrm{mm}$ and $h_{a}=\{0.20,0.40,0.60\} \mathrm{mm}$ (with fixed dimensions of $W=10.67 \mathrm{~mm}, p=3.6 \mathrm{~mm}$ and $\left.W_{p}=1.88 \mathrm{~mm}\right)$. Next step has been the computation of the electromagnetic fields in HFSS. Finally, a diffusion type problem for the evolution of the electron density has been solved by the commercial software tool SPARK3D ${ }^{\circledR 2}$, where the electromagnetic fields obtained in HFSS have been used as input data. The power breakdown limit has been simulated for dry air as gas and for a temperature of $293 \mathrm{~K}$ in the whole pressure range (between 1 mbar and 1000 mbar). Peak power values for multiple frequencies in the stopband region of each design have been computed.

In order to simplify the results, Fig. 4 shows the obtained power threshold values at different frequencies for a pressure set to 600 mbar, which is considered to be within the high pressure region. Parameter $h_{a}$ has been fixed for the plotted curves in each graph, and each color represents a different

${ }^{2}$ SPARK3D, Copyright (C) 2012-2013 AuroraSat (now Dassault Systèmes), available on http://www.cst.com/products/spark3d 


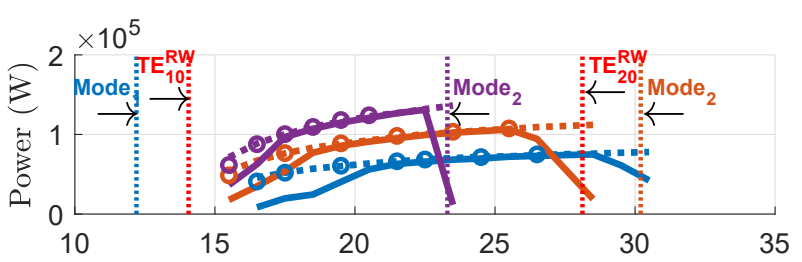

(a)

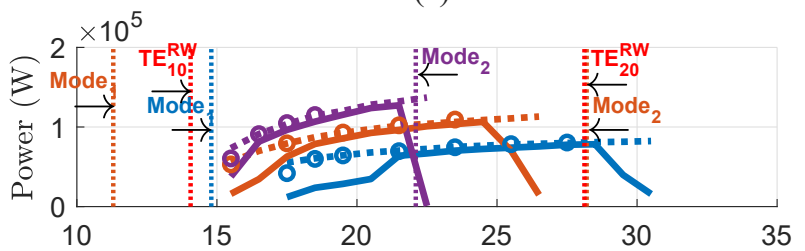

(b)

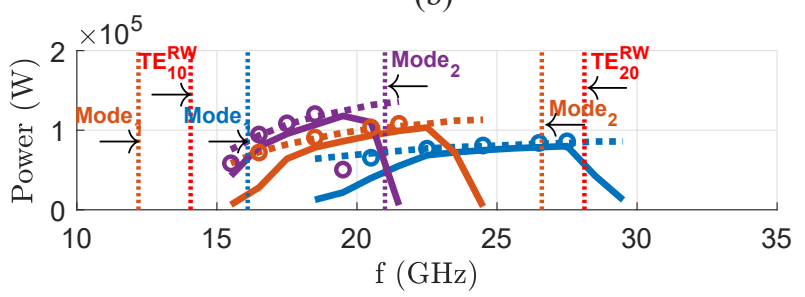

(c)

\begin{tabular}{|c|}
\hline GGW $\left(h_{p}=3.00 \mathrm{~mm}\right)$ \\
$\cdots \mathrm{RW}\left(h=3.00+h_{a} \mathrm{~mm}\right)$ \\
GGW $\left(h_{p}=4.50 \mathrm{~mm}\right)$ \\
RW $\left(h=4.50+h_{a} \mathrm{~mm}\right)$ \\
GGW $\left(h_{p}=6.00 \mathrm{~mm}\right)$ \\
$\ldots \mathrm{RW}\left(h=6.00+h_{a} \mathrm{~mm}\right)$ \\
\hline
\end{tabular}

Fig. 4. Power limit due to corona discharge breakdown for different frequencies at pressure of 600 mbar. Values for RW (dashed line) and GGW (solid line) are plotted for comparison. Vertical dotted lines are included to indicate the cutoff frequencies of $\mathrm{TE}_{10}^{R W}$ and $\mathrm{TE}_{20}^{R W}$, and also the stopband region for each design. Please note that unrepresented modes are out of the band shown between $10 \mathrm{GHz}$ and $35 \mathrm{GHz}$. Circles symbolize the enhanced power limit with the design criterion presented in Section III. (a) $h_{a}=0.20 \mathrm{~mm}$. (b) $h_{a}=0.40 \mathrm{~mm}$. (c) $h_{a}=0.60 \mathrm{~mm}$.

value of $h_{p}$. Power limits for the equivalent rectangular waveguides (dashed lines), where $h_{R W}=h_{p}+h_{a}$, are also represented for comparison with groove gap waveguide values (solid lines).

Some comments can be arisen from the results shown in Fig. 4:

- Higher values of $h_{p}$, for fixed $h_{a}$, lead to shifting down the stopband behavior, and therefore, higher power breakdown limits happen, as also occurs in rectangular waveguides as $h_{R W}$ increases.

- Lower values of $h_{a}$, for fixed $h_{p}$, lead to wider stopband bandwidths.

- GGW power breakdown is more like RW power breakdown at the high part of the stopband, due to the decrease of the electric field secondary lobes as seen in the previous section.

\section{StRATEGy FOR ENHANCING THE PPHC}

Throught the study performed in Section II, it is clear that peak power limits for GGW differ from those of RW, especially at the lower frequencies of the stopband, where an

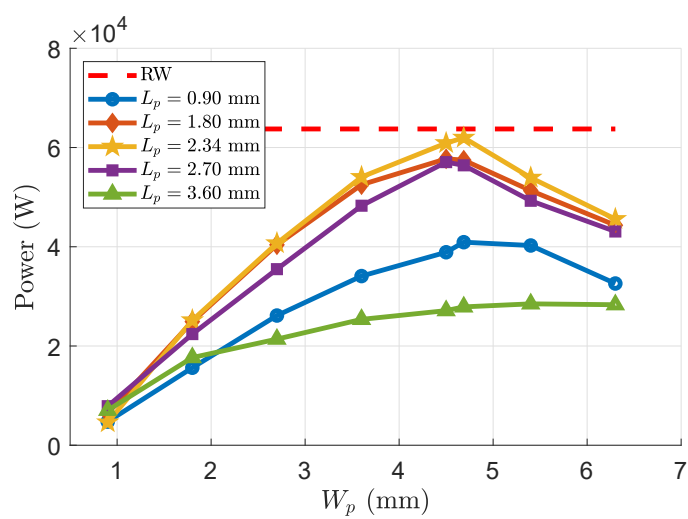

(a)

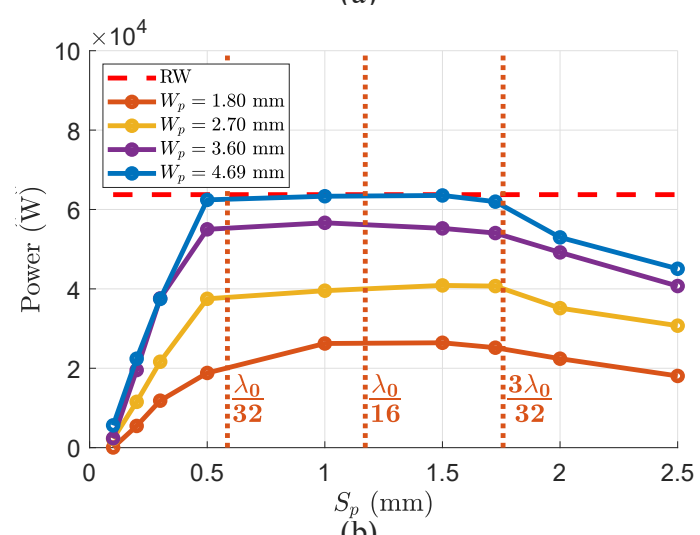

(b)

Fig. 5. Power limit at 600 mbar and $f=16 \mathrm{GHz}$. (a) Study of $W_{p}$ and $L_{p}$. (b) Study of $S_{p}$

unconfined quasi-TE 10 mode results in a strong electric field over the first lateral row of pins.

With the aim of enhancing the PPHC in GGW, mainly at lower frequencies in the stopband region, a proposed strategy will be presented below. The main idea for achieving this improvement is the adjustment of the three geometric pin dimensions (see Fig. 1(b)): length $L_{p}$ (along Z-axis), width $W_{p}$ (along X-axis) and pin separation $S_{p}$ (along both Z- and $\mathrm{X}$-axes). Please note that the periodicity along $\mathrm{Z}$-axis is now $p_{z}=L_{p}+S_{p}$, and along $\mathrm{X}$-axis is $p_{x}=W_{p}+S_{p}$. Even though the periodic structure of the bed of nails has usually been realized by square pins $\left(L_{p}=W_{p}\right)$, the suggested strategy proposes the design of rectangular pins.

\section{A. Study of $W_{p}$ and $L_{p}$}

Several layouts as shown in Fig. 1(a) have been designed for multiple combinations of $W_{p}=\{0.90,1.80,2.70,3.60,4.50,4.69,5.40,6.30\} \mathrm{mm}$ and $L_{p}=\{0.90,1.80,2.34,2.70,3.60\} \mathrm{mm}$ (with fixed dimensions of $W=10.67 \mathrm{~mm}, h_{p}=4.50 \mathrm{~mm}, h_{a}=0.40 \mathrm{~mm}$ and $S_{p}=1.72 \mathrm{~mm}$ ). SPARK3D ${ }^{\circledR}$ has been used to obtain the power breakdown at 600 mbar and at the operating frequency of $f=16 \mathrm{GHz}$, where secondary lobes of $\left|\hat{E}_{y}\right|$ appear above the pins, as shown in Fig. 3(b).

The obtained results are shown in Fig. 5(a), where the power limit is represented as a function of $W_{p}$, and various curves are plotted to represent different values of $L_{p}$. As we can 


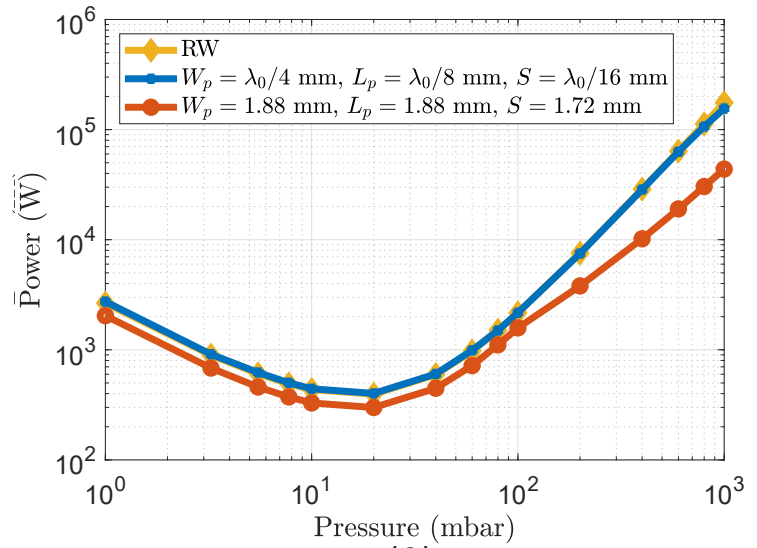

(a)

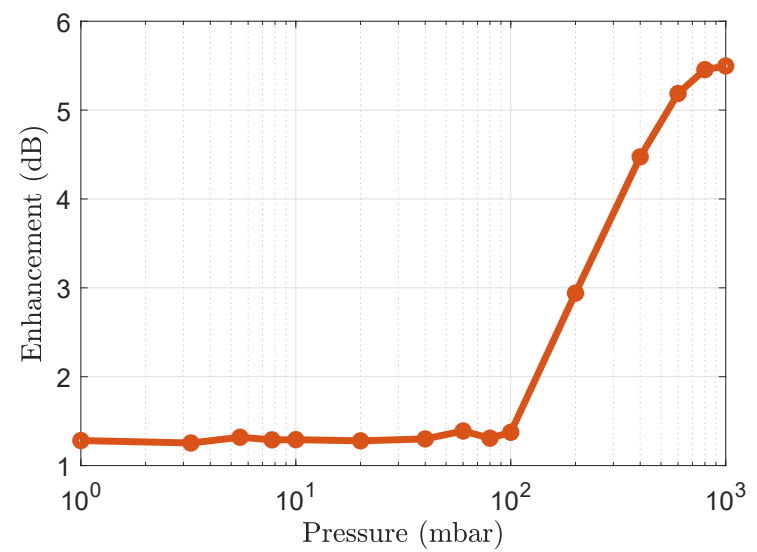

(b)

Fig. 6. (a) Corona discharge breakdown (Paschen curves). (b) Enhancement between optimized and non-optimized designs. $f=16 \mathrm{GHz}$, $h_{p}=4.50 \mathrm{~mm}$ and $h_{a}=0.40 \mathrm{~mm}$.

observe, PPHC reaches a value similar to the equivalent RW when $W_{p}=4.69 \mathrm{~mm} \approx \lambda_{0} / 4$ and $L_{p}=2.34 \mathrm{~mm} \approx \lambda_{0} / 8$. From these results, one could assume these dimensions as a first approximation of a design criterion for optimizing peak power in GGW.

\section{B. Study of $S_{p}$}

Finally, the pin separation is other possible parameter for studying PPHC variation in groove gap waveguides. Multiple designs have now been simulated for different values of $S_{p}$ and $W_{p}$, where the previous optimized dimension $L_{p}=\lambda_{0} / 8$ has been fixed.

Fig. 5(b) shows the results obtained by SPARK3D ${ }^{\circledR}$. One can see that small separations are not desirable by the fact that high electric field density could appear between pins. Furthermore, electric field could be less confined for higher values of $S_{p}$. So, we can see that the peak power is maximum for values of $S_{p}$ between $\lambda_{0} / 32$ and $3 \lambda_{0} / 32$. Therefore, the last design criterion could be a centered value of $S_{p}=\lambda_{0} / 16$.

\section{Results}

After carrying out the above studies, optimized dimensions of $W_{p}, L_{p}$ and $S_{p}$ have been obtained to maximize the PPHC in GGW. However, the previous results have been simulated

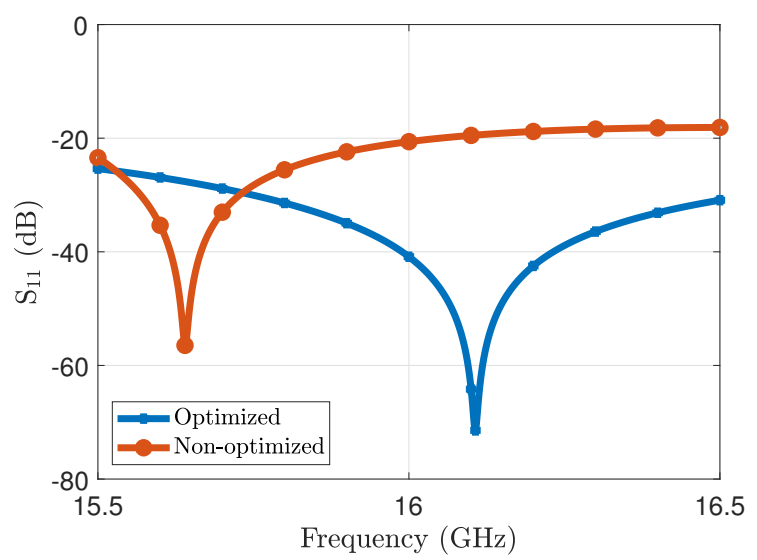

Fig. 7. Reflection coefficient comparison between the optimized design $\left(W_{p}=\lambda_{0} / 4 \mathrm{~mm}, L_{p}=\lambda_{0} / 8 \mathrm{~mm}, S_{p}=\lambda_{0} / 16 \mathrm{~mm}\right)$ and the nonoptimized design $\left(W_{p}=1.88 \mathrm{~mm}, L_{p}=1.88 \mathrm{~mm}, S_{p}=1.72 \mathrm{~mm}\right.$ )

for a single frequency and fixed dimensions of $h_{p}$ and $h_{a}$, so it becomes necessary to extend the simulations of the proposed strategy to other dimension possibilities.

In Fig. 4, the enhanced power limit for multiple designs and frequencies, where the design criterion is used, are plotted with circles. One can see that, in most cases, the improved power limit for a GGW is similar to the equivalent RW, specially for lower values of $h_{p}$ and $h_{a}$ where the use of the design strategy makes the most sense. It is also important to highlight that the stopband bandwidth is narrower when the proposed strategy is applied (mainly, the upper cut-off stopband frequency is shifted down), so it explains the decrease of power threshold in the plotted example $h_{p}=6 \mathrm{~mm}, h_{a}=0.6 \mathrm{~mm}$ and $f=19.5$ $\mathrm{GHz}$ (see Fig. 4c), where the upper limit of the stopband is exceeded. For this reason, it is necessary to reach a trade-off between the improvement of PPHC and the reduction of the stopband bandwidth. Therefore, the use of the design criterion becomes advisable at lower frequencies in the stopband region, with the main purpose of decreasing secondary lobes above the first lateral row of pins.

From the designer's point of view, it would be first important to select suitable dimensions of $h_{p}$ and $h_{a}$ for fixing the desired bandwidth where the operating quasi-TE 10 mode is able to be propagated. Next, a fine adjustment applying the proposed design strategy of $W_{p}, L_{p}$ and $S_{p}$ would be desirable for enhancing corona discharge breakdown.

In order to validate the suggested strategy in the whole pressure range, the Paschen curves are represented in Fig. 6(a) for an optimized (blue line) and non-optimized (red line) pin dimensions. As we can see in Fig. 6(b), the improvement of power level thresholds between both designs is around $1.2 \mathrm{~dB}$ for low and middle pressures, while it is increased to reach a maximum value of $5.5 \mathrm{~dB}$ at 1000 mbar (sea level pressure). In Fig. 7, the reflection coefficient is represented for a waveguide bandwidth of $1 \mathrm{GHz}$ for both designs.

Finally, the electric field distribution for both cases is shown in Fig. 8. One can see that maximum values are located in the air gap above the first lateral row of pins for the non-optimized design, so it leads to lower PPHC than the optimized design. 

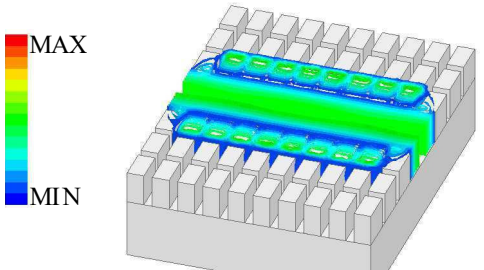

(a)

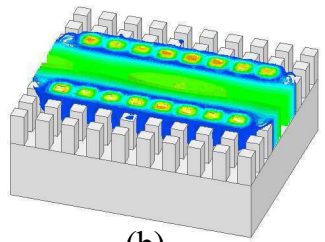

(b)
$0 \mathrm{~mm} \quad 30 \mathrm{~mm}$

Fig. 8. Electric field distribution at $f=16 \mathrm{GHz}$. (a) Optimized. (b) Nonoptimized.

In order to validate the proposed solution, a campaign is planned at the European High-Power RF Space Laboratory (Valencia, Spain), and the experimental results are expected to be presented in the conference.

\section{CONCLUSIONS}

In this paper, a new design strategy for improving PPHC in groove gap waveguides has been proposed. First of all, the investigation has been focused on the study of the quasi$\mathrm{TE}_{10}$ mode distribution, where the results show that maximum electric field is located in the air gap, above the first lateral row of pins, at lower frequencies of the stopband region. Next, the study of PPHC for different pin geometric dimensions has indicated that, in GGW it is lower than that of RW when secondary lobes appear for lower frequencies. Finally, a study of the parameters $W_{p}, L_{p}$ and $S_{p}$ has been carried out to obtain the optimal dimensions for enhancing peak power limits. The simulation results have shown a substantial improvement without degrading the electrical response by using the proposed strategy.

\section{ACKNOWLEDGMENT}

This work has been supported by the University of Alicante through the fellowship grant UAFPU2018-054 and by the "Ministerio de Ciencia e Innovación" through research projects PID2019-103982RB-C41 and PID2019-103982RB-C43,

\section{REFERENCES}

[1] A. D. MacDonald, Microwave breakdown in gases. New York: Wiley, 1966.

[2] C. Ernst and V. Postoyalko, "Prediction of peak internal fields in directcoupled-cavity filters," IEEE Transactions on Microwave Theory and Techniques, vol. 51, no. 1, pp. 64-73, January 2003.

[3] C. Vicente, M. Mattes, D. Wolk, B. Mottet, H. Hartnagel, J. Mosig, and D. Raboso, "Microwave breakdown prediction in rectangular waveguide based components," in Proceedings of the German Microwave Conferece, 2005, pp. 17-20.

[4] T. Pinheiro-Ortega, J. Monge, S. Marini, J. Sanz, E. Sorolla, M. Mattes, C. Vicente, J. Gil, V.E. Boria, and B. Gimeno, "Microwave corona breakdown prediction in arbitrarily-shaped waveguide based filters," IEEE Microwave and Wireless Components Letters, vol. 20, no. 4, pp. 214-216, April 2010.

[5] F. J. P. Soler, S. Anza, M. Mattes, C. M. España, F. Quesada, M. Jiménez, J. Gil, C. Vicente, J.R. Mosig, D. Raboso, V. E. Boria, B. Gimeno, and A. Alvarez-Melcon, "Rigorous investigation of RF breakdown effects in high power microstrip passive circuits," in 2009 IEEE MTT-S International Microwave Symposium Digest, June 2009, pp. 833-836.
[6] M. Sánchez-Soriano, Y. Quéré, V. Le Saux, S. Marini, M.S. Reglero, V.E. Boria, and C. Quendo, "Peak and average power handling capability of microstrip filters," IEEE Transactions on Microwave Theory and Techniques, vol. 67, no. 8, pp. 3436-3448, Aug 2019.

[7] A. Morales-Hernandez, M. Sánchez-Soriano, S. Marini, V.E. Boria, and M. Guglielmi, "Cover-ended resonators to increase corona discharge thresholds in microstrip bandpass filters," in 50th European Microwave Conference (EuMC 2020), in press 2020.

[8] M. Baquero-Escudero, A. Valero-Nogueira, M. Ferrando-Rocher, B. Bernardo-Clemente, and V. E. Boria-Esbert, "Compact combline filter embedded in a bed of nails," IEEE Transactions on Microwave Theory and Techniques, vol. 67, no. 4, pp. 1461-1471, April 2019.

[9] M. Ferrando-Rocher, A. Valero-Nogueira, J. I. Herranz-Herruzo, A. Berenguer, and B. Bernardo-Clemente, "Groove gap waveguides: A contactless solution for multilayer slotted-waveguide array antenna assembly," in 2016 10th European Conference on Antennas and Propagation (EuCAP), 2016, pp. 1-4.

[10] E. Rajo-Iglesias, M. Ferrando-Rocher, and A. U. Zaman, "Gap waveguide technology for millimeter-wave antenna systems," IEEE Communications Magazine, vol. 56, no. 7, pp. 14-20, July 2018.

[11] E. Rajo-Iglesias and P.-S. Kildal, "Numerical studies of bandwidth of parallel-plate cut-off realised by a bed of nails, corrugations and mushroom-type electromagnetic bandgap for use in gap waveguides," IET Microwaves, Antennas Propagation, vol. 5, no. 3, pp. 282-289, February 2011

[12] A. Berenguer, V. Fusco, D.E. Zelenchuk, D. Sánchez-Escuderos, M. Baquero-Escudero, and V.E. Boria-Esbert, "Propagation characteristics of groove gap waveguide below and above cutoff," IEEE Transactions on Microwave Theory and Techniques, vol. 64, no. 1, pp. 27-36, January 2016. 